\title{
PREENCHIMENTO DA CADERNETA DE SAÚDE DA CRIANÇA NA PRIMEIRA INFÂNCIA
}

\section{Completion of the child health record book in early childhood Relleno de la libreta de salud del niño de primera infancia}

\author{
Jeanne Lúcia Gadelha Freitas iD \\ Fundação Universidade Federal de Rondônia - UNIR - Porto Velho (RO) - Brasil \\ Priscilla Perez da Silva Pereira (iD \\ Fundação Universidade Federal de Rondônia - UNIR - Porto Velho (RO) - Brasil \\ Kátia Fernanda Alves Moreira iD \\ Fundação Universidade Federal de Rondônia - UNIR - Porto Velho (RO) - Brasil \\ Nathalia Halax Orfão iD \\ Fundação Universidade Federal de Rondônia - UNIR - Porto Velho (RO) - Brasil \\ Daniela Ferreira Borba Cavalcante iD \\ Fundação Universidade Federal de Rondônia - UNIR - Porto Velho (RO) - Brasil
}

Rayanne Cavalcante Nascimento iD

Secretaria Municipal de Saúde - SEMUSA - Costa Marques (RO) - Brasil

Tatiane Maciel Mendes iD

Secretaria Municipal de Saúde - SEMUSA - Guajará-Mirim (RO) - Brasil

Águida Thomaz Santos (iD

Secretaria Municipal de Saúde - SEMUSA - Guajará-Mirim (RO) - Brasil

\section{RESUMO}

Objetivo: Avaliar o preenchimento da Caderneta de Saúde da Criança em uma capital da Região Norte do Brasil. Métodos: Estudo transversal e descritivo, realizado num hospital pediátrico de uma capital na Região Norte, com 420 crianças com idade inferior a cinco anos. A coleta de dados ocorreu no período de abril a outubro de 2017 , em entrevista com o cuidador principal da criança, na qual se questionou informações referentes ao cuidador, à criança e orientações recebidas sobre a caderneta, bem como o seu preenchimento, utilizando-se um sistema de escore. A análise foi realizada por meio de estatística descritiva e cálculo da Razão de Prevalência, por meio de Regressão de Poisson, no software Stata ${ }^{\circledR}$, versão 13. Resultados: Apenas $25,5 \%(n=111)$ das Cadernetas de Saúde da Criança tiveram preenchimento satisfatório. O preenchimento não satisfatório esteve associado $(p=0,01)$ a casos em que o cuidador principal não era os pais ou avós, o cuidador possuía escolaridade menor que nove anos, a mãe não havia realizado o pré-natal ou o acompanhamento pelo Sistema Único de Saúde, e entre os cuidadores que não receberam orientações sobre a importância da caderneta. O maior índice de preenchimento da caderneta foi referente às vacinas aplicadas $(99,3 \%, n=417)$, e um dos menores índices foi sobre o desenvolvimento neuropsicomotor $(18,1 \%, n=202)$. Conclusão: O preenchimento insatisfatório da Caderneta de Saúde da Criança mostra as fragilidades no acompanhamento do crescimento e desenvolvimento integral, principalmente na primeira infância.

Descritores: Saúde da Criança; Vigilância em Saúde Pública; Crescimento e Desenvolvimento.

\section{ABSTRACT}

Objective: To evaluate the completion of the Child Health Record Book in a capital city in Northern Brazil. Methods: A descriptive cross-sectional study was carried out in a pediatric hospital in a capital city in the Northern Region with 420 children under the age of five. Data collection took place from April to October 2017 through interviews with the primary caregiver of the child. Information

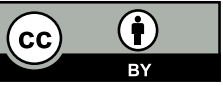


on the caregiver, the child and access to guidelines on the record book and its completion were collected using a score system. The analysis was performed using descriptive statistics and calculating Prevalence Ratio using Poisson Regression in Stata ${ }^{\circledR}$ version 13. Results: Only 25.5\% of the Child Health Record Books were satisfactorily completed. Unsatisfactory completion was associated $(p=0.01)$ with children whose primary caregiver was not a parent or grandparent, caregivers with less than nine years of study, mothers who had not undergone prenatal care or been followed up by the Unified Health System and caregivers who did not have access to guidelines on the importance of the record book. The highest rate of completion of the record book was related to vaccines received $(99.3 \%, n=417)$ and one of the lowest rates was related to neuro-psychomotor development (18.1\%, $n=202)$. Conclusion: The unsatisfactory completion of the Child Health Record Book shows weaknesses in monitoring the growth and integral development, especially in early childhood.

Descriptors: Child Health; Public Health Surveillance; Growth and Development.

\section{RESUMEN}

Objetivo: Evaluar el relleno de la Libreta de Salud del Niño de una capital de la Región Norte de Brasil. Métodos: Estudio transversal y descriptivo realizado en hospital pediátrico de una capital de la Región Norte con 420 niños con menos de cinco años de edad. La recogida de datos se dio en el periodo entre abril y octubre de 2017 a través de entrevista con el cuidador principal del niño en la cual se ha preguntado informaciones sobre el cuidador, el niño y las orientaciones que han recibido sobre la libreta así como su relleno, utilizándose un sistema de puntuación. El análisis ha sido realizado por estadística descriptiva y el cálculo de la razón de prevalencia a través de la regresión de Poisson con el software Stata ${ }^{\circledR}$, la versión 13. Resultados: Solamente el 25,5\% $(n=111)$ de las Libretas de Salud del Niño presentaron relleno satisfactorio. El relleno no satisfactorio se asoció $(p=0,01)$ con los casos en los cuales el cuidador principal no era uno de los padres o abuelos, el cuidador tenía menos de nueve años de escolaridad, la madre no había hecho el prenatal o el seguimiento por el Sistema Único de Salud y entre los cuidadores que no han recibido orientaciones sobre la importancia de la libreta. El mayor índice de relleno de la libreta ha sido sobre las vacunas aplicadas $(99,3 \%, n=417)$ y uno de los más bajos índices ha sido sobre el desarrollo neuropsicomotor $(18,1 \%, n=202)$. Conclusión: El relleno insatisfactorio de la Libreta de Salud del Niño señala las fragilidades del seguimiento del crecimiento y desarrollo integral, en especial de la primera infancia.

Descriptores: Salud del Niño; Vigilancia en Salud Pública; Crecimiento y Desarrollo.

\section{INTRODUÇÃO}

A avaliação do crescimento e desenvolvimento infantil é um dos pilares da vigilância à saúde da criança visando à redução da mortalidade infantil em todo mundo( ${ }^{(1,2)}$. Estratégia instituída na metade do século passado, tornou-se um eixo fundamental da atenção à saúde da criança, pois permite uma avaliação apropriada e oportuna para monitorar os indicadores associados às ações de prevenção da morbimortalidade nos primeiros anos de vida(3).

No mundo, cerca de 5,9 milhões de crianças com até cinco anos morreram por doenças evitáveis em 2015, e serão mais 6,9 milhões de óbitos infantis até $2030^{(4)}$. No Brasil, somente em 2016, morreram 35.619 crianças com até cinco anos, $58 \%$ de causas preveníveis pela vigilância sistemática da saúde materno-infantil(5). Nos últimos cinco anos, a prioridade mundial tem sido os chamados " 1.000 dias críticos" da criança, ou seja, dos nove meses de vida fetal até os dois anos de nascimento, período de extrema vulnerabilidade à sobrevivência e desenvolvimento infantil( ${ }^{(6)}$.

Nesse cenário, o acompanhamento do crescimento e desenvolvimento feito por meio da Caderneta de Saúde da Criança (CSC) é crucial na prevenção da morbimortalidade ${ }^{(7)}$ já que permite a identificação precoce de agravos ${ }^{(8,9)}$. Países desenvolvidos exibem menores taxas de mortalidade infantil devido à utilização de cadernetas de saúde da mãe/filho, por conectar os dois aos serviços de saúde do país ${ }^{(1,2)}$. Mesmo em países com baixa cobertura de serviços de saúde, a experiência de registros sistemáticos do binômio mãe/filho em cadernetas promoveu a aquisição de cuidados contínuos, desde a gestação até a entrada da criança na escola, com taxa de seguimento de $70,2 \%$ nas áreas acompanhadas ${ }^{(10)}$.

No Brasil, a atual CSC é considerada passaporte de cidadania infantil, pois possibilita todos os registros dos cuidados de saúde da criança de zero até dez anos de idade. Sua primeira versão, publicada em 2005, substituiu o cartão da criança, em que se fazia a supervisão do crescimento e imunização. Nas cinco edições subsequentes, a CSC incorporou dados significativos, como o acompanhamento de criança com síndrome de Down e autismo, gráficos de índice de massa corporal e pressão arterial|(11,12).

A vigilância do desenvolvimento infantil é efetivada há 32 anos, porém há escassez de estudos e descaso generalizado dos registros na CSC, uma vez que o seu percentual de preenchimento não ultrapassa $30,4 \%{ }^{(3)}$. As causas vão desde o preenchimento incorreto/incompleto até a ausência de avaliação do desenvolvimento 
neuropsicomotor e do crescimento ${ }^{(13,14)}$. A fragilidade dos registros da CSC pode ter relação com a falta de capacitação dos profissionais ${ }^{(15)}$ ou com o número insuficiente de equipes de saúde ${ }^{(16)}$. Além disso, o desconhecimento dos pais sobre a importância da CSC $^{(17,18)}$ reforça a pouca atenção ao preenchimento dela pela própria sociedade, que ainda não se interessa pelo assunto ${ }^{(3)}$.

Em uma busca nas principais bases eletrônicas indexadoras de estudos nessa área não foram encontradas pesquisas sobre o preenchimento da CSC no estado de Rondônia, necessitando-se conhecer a realidade e uso dessa ferramenta na atenção à saúde da criança. Diante de tais aspectos, da experiência em campos de prática em puericultura, com ausência de registros na CSC em quase todos os seus itens, e do desconhecimento de sua importância por profissionais e cuidadores das crianças, verbalizado como um "cartão de vacinas", pretendeu-se avaliar o preenchimento da Caderneta de Saúde da Criança em uma capital da Região Norte do Brasil.

\section{MÉTODOS}

Trata-se de um estudo transversal e descritivo, realizado entre os meses de abril a outubro de 2017, em um hospital pediátrico na cidade de Porto Velho, Rondônia, Brasil, uma instituição de referência da região de saúde Madeira-Mamoré, que compreende cinco municípios, possui IDH de 0,736 e população de 519.436 habitantes, sendo 51.292 crianças menores de cinco anos, ou seja, 9,8\% da população residente nesse município ${ }^{(19)}$.

É o único estabelecimento de saúde do Sistema Único de Saúde (SUS) da capital com atendimento pediátrico em nível ambulatorial, urgência e emergência. Em 2015, o hospital atendeu 56.370 crianças até 12 anos, principalmente no que concerne a doenças respiratórias $(31,7 \%)$, trauma $(14,8 \%)$, doenças do aparelho digestivo $(8,4 \%)$ e geniturinário $(6,7 \%)$, e doenças infecto-parasitárias $(3,9 \%)^{(20)}$.

A população do estudo foi constituída por cuidadores de crianças com menos de cinco anos de idade e que portavam a CSC no momento da coleta de dados. O tamanho amostral baseou-se no total de 2.483 crianças, até cinco anos, atendidas no referido hospital em 2015(20). Realizou-se o cálculo amostral por meio do EPI INFO 3.5.2, considerando erro de $5 \%$, intervalo de confiança de $95 \%$ e prevalência de $50 \%$ do preenchimento satisfatório da CSC, considerando que não foram encontrados estudos sobre a temática na região Norte para que a prevalência fosse utilizada no cálculo. A amostra prevista era de 333 participantes, contudo, considerando-se um acréscimo de $20 \%$ para perdas e recusas, obteve-se uma amostra final de 399 crianças.

Os critérios de inclusão compreenderam a presença do familiar ou cuidador principal, com idade superior a 18 anos, acompanhando crianças de até cinco anos, hospitalizados ou aguardando atendimento, e que estivessem com a CSC no momento da entrevista. Os critérios de exclusão foram crianças hospitalizadas em estado grave na Unidade de Terapia Intensiva ou na Unidade de Cuidados Intermediários.

Realizou-se a coleta de dados na sala de espera do hospital, enquanto os cuidadores e as crianças aguardavam atendimento pela equipe multiprofissional. A coleta ocorreu por meio de uma entrevista estruturada, realizada com o principal cuidador da criança, e pela análise dos registros da CSC. Para a entrevista, utilizou-se um roteiro elaborado pelos autores, composto por 47 perguntas distribuídas em três tópicos: caracterização do cuidador, características da criança e preenchimento da CSC. Foi realizado previamente o teste piloto e a capacitação dos pesquisadores.

Para análise do preenchimento da CSC no que se refere aos registros de crescimento e desenvolvimento, utilizou-se um sistema de escore baseado em dois estudos brasileiros sobre o preenchimento da $\operatorname{CSC}^{(21,22)}$. O sistema atribui o valor de um ponto para itens preenchidos corretamente e zero para aqueles preenchidos incorretamente ou não preenchidos. Após somatória dos valores, os escores foram definidos como preenchimento insatisfatório quando menos de $60 \%$ dos itens eram preenchidos (<14 pontos) e como preenchimento satisfatório quando mais de $60 \%$ dos itens estavam preenchidos ( $\geq 14$ pontos). A pontuação máxima que cada CSC poderia obter era de 21 pontos, sendo que cada ponto equivalia a $4,8 \%$ de preenchimento da $\operatorname{CSC}^{(21,22)}$.

Neste estudo, o desfecho de interesse compreendeu o preenchimento satisfatório da CSC. As variáveis estudadas foram referentes ao principal cuidador, à criança e sobre as orientações recebidas pelo cuidador em relação à importância da CSC. No caso das características do cuidador principal da criança: cuidador principal (pai ou mãe; avô ou avó; outra pessoa); sexo (feminino ou masculino); idade ( $\geq 20$ a 29 anos; > 30 anos; <20); estado marital (com companheiro ou sem companheiro) escolaridade ( $\geq 9$ anos ou $<9$ anos), trabalho remunerado (não ou sim), renda familiar ( $>2$ salários mínimos ou $\leq 2$ salários mínimos), realização do pré-natal (sim ou não/não sabe) e local do pré-natal (SUS ou plano de saúde particular). No caso das características da criança: idade da criança ( $\geq 1$ ano; $\geq 6$ meses a 1 ano; $<6$ meses), sexo da criança (masculino ou feminino), peso ao nascer ( $\geq 2500$ gramas ou $<2500$ 
gramas, peso limítrofe utilizado pelo Ministério da Saúde(11) para classificação de peso de baixo peso ao nascer) e comprimento ao nascer ( $\geq 47$ centímetros ou $<47$ centímetros). Já em relação às orientações sobre a importância da CSC: recebeu orientações sobre a CSC (sim ou não), profissionais que realizaram as orientações (enfermeiro; outro profissional de nível superior; nível médio).

Realizou-se análise descritiva dos dados e, para análise da associação entre as variáveis, utilizaram-se os testes qui-quadrado de Pearson e Exato de Fisher. Como medida de efeito entre o preenchimento satisfatório da CSC e as variáveis investigadas, aplicaram-se cálculos de Razão de Prevalência (RP) e Intervalos de Confiança de 95\% (IC 95\%) por meio de regressão de Poisson. Os dados foram processados no software Stata ${ }^{\circledR}$ (College Station, Texas, USA) versão 13.

O presente estudo está vinculado ao projeto matriz "Avaliação da Atenção à Saúde da Criança em Porto Velho - RO", do Centro de Estudos e Pesquisa em Saúde Coletiva da Fundação Universidade Federal de Rondônia. O estudo cumpriu os aspectos éticos da Resolução 466/12 do Conselho Nacional de Saúde(23). Os entrevistados participaram voluntariamente do estudo, mediante assinatura de Termo de Consentimento Livre e Esclarecido. Ademais, foram assegurados os princípios de autonomia, anonimato e não maleficência aos envolvidos. Este estudo recebeu aprovação pelo Comitê de Ética e Pesquisa sob o Protocolo nº 1.849.757.

\section{RESULTADOS}

Dos 564 cuidadores, $89,0 \%(n=502)$ foram entrevistados, pois tinham a CSC no momento da coleta. Desse total, $19,5 \%$ ( $n=98$ ) recusaram ou não concluíram a entrevista, totalizando 420 participantes. O motivo principal de recusa ou inconclusão foi a criança necessitar de avaliação médica no momento da abordagem e o cuidador não mais desejar continuar a entrevista para atender algum familiar no celular ou por não saber responder algumas perguntas.

A maioria dos cuidadores era do sexo feminino $(97,4 \%, n=409)$, idade média de 29,2 anos $( \pm 8,2)$, com companheiro $(74,0 \%, n=311)$, escolaridade superior a nove anos de estudo $(93,8 \%, n=394)$, não possuía trabalho remunerado $(65,7 \%, n=276)$ e renda familiar abaixo de dois salários mínimos $(74,0 \%, n=311)$. A maioria das mães havia realizado pré-natal $(98,3 \%, n=413)$ e o acompanhamento foi, sobretudo, no SUS $(86,7 \%, n=358)$ (Tabela I).

Com relação às crianças, mais da metade tinha um ano $(71,7 \%, n=302$; média 1,9 e $\pm 1,9)$, sexo masculino $(52,4 \%, n=220)$, peso ao nascer acima de 2.500 gramas $(92,9 \%, n=288)$ e comprimento ao nascer de 47 centímetros $(93,5 \%, n=274)$. No que concerne às orientações sobre a CSC, um pouco mais da metade dos cuidadores $(51,9 \%$, $\mathrm{n}=218$ ) afirmou que, pelo menos uma vez, já haviam recebido orientações sobre a CSC, principalmente do enfermeiro $(69,3 \%, n=151$; Tabela I).

As variáveis relacionadas ao principal cuidador - sexo, idade, estado marital, tipo de trabalho e renda familiar - não foram associados ao desfecho. Da mesma maneira, as características das crianças - idade, sexo, peso ao nascer e estatura ao nascer e o profissional que realizou orientações da CSC - também não apresentaram associação estatisticamente significativa com o preenchimento satisfatório da CSC (Tabela I).

Entre as características analisadas, verificou-se que o preenchimento insatisfatório da CSC esteve associado às crianças cujo cuidador principal não era pais ou avós (RP 1,36; IC 95\% 1,29; 1,45), o cuidador tinha menos de nove anos de estudo ( $\operatorname{RP} 1,28$; IC 95\% 1,12; 1,45), cuja mãe não realizou o pré-natal ( $\operatorname{RP} 1,37 ;$ IC $95 \% 1,29 ; 1,45)$, o pré-natal não foi feito no SUS (RP 1,23; IC 95\% 1,09; 1,39) ou cujos cuidadores não receberam orientações sobre a CSC (RP 1,32; IC 95\% 1,17; 1,48).

A Tabela II apresenta o percentual de preenchimento satisfatório da CSC conforme itens avaliados. Das 420 cadernetas avaliadas, apenas $25,5 \%(n=111)$ tinham preenchimento satisfatório, ou seja, possuíam pelo menos $60 \%$ dos registros com variação de 14 a 21 pontos.

Do total de registros sobre gravidez, parto e puerpério preenchidos na CSC, houve maior frequência de preenchimento para tipo de parto $(55,5 \%, n=233)$ e dados do parto $(45,2 \%, n=190)$, com menor frequência para registros sobre a gravidez $(31,7 \%, n=133)$ e o puerpério $(21,2 \%, n=89$; Tabela II). Sobre o recém-nascido, houve maior preenchimento da data do nascimento $(75 \%, n=315)$ e do peso ao nascer $(74,3 \%, n=312)$, e menor para APGAR $(64,8 \%, n=272)$ e teste do pezinho $(19,5 \%, n=82)$. Nos registros do crescimento e desenvolvimento da criança, houve maior percentual sobre o peso $(36,9 \%, n=154)$ e comprimento/altura $(35,0 \%, n=147)$, e menores percentuais para curva de crescimento do perímetro cefálico $(21,9 \%, n=92)$ e avaliação do desenvolvimento neuropsicomotor $(18,1 \%, n=202)$. Em relação às informações adicionais, o item registro de vacinação teve maior preenchimento $(99,3 \%, \mathrm{n}=417)$. 
Tabela I - Características do principal cuidador, da criança e sobre orientações recebidas pelo cuidador em relação à Caderneta de Saúde da Criança, Hospital Infantil Cosme e Damião. Porto Velho, Rondônia, Brasil, 2017 (n=420).

\begin{tabular}{|c|c|c|c|c|c|}
\hline \multirow{2}{*}{ Variável } & \multicolumn{2}{|c|}{ Total } & \multirow{2}{*}{$\begin{array}{l}\text { Preenchimento satisfatório da CSC } \\
\qquad \%(n=111)\end{array}$} & \multirow{2}{*}{ RP (IC95\%) } & \multirow{2}{*}{$\mathbf{p}$} \\
\hline & $\mathrm{n}$ & $\%$ & & & \\
\hline \multirow{2}{*}{\multicolumn{6}{|c|}{ Características do principal cuidador }} \\
\hline \multicolumn{3}{|l|}{ Cuidador principal } & & & \\
\hline Pai ou mãe & 392 & 93,3 & 94,6 & 1 & \\
\hline Avô ou avó & 25 & 6,0 & 5.4 & $1,04(0,82 ; 1,30)$ & 0,75 \\
\hline Outra pessoa & 3 & 0,7 & 0 & $1,36(1,29 ; 1,45)$ & 0,01 \\
\hline \multicolumn{6}{|l|}{ Sexo do cuidador principal } \\
\hline Feminino & 409 & 97,4 & 98,2 & 1 & \\
\hline Masculino & 11 & 2,6 & 1,80 & $1,11(0,84 ; 1,48)$ & 0,45 \\
\hline \multicolumn{6}{|l|}{ Idade do cuidador principal } \\
\hline$\geq 20$ a 29 anos & 235 & 56,0 & 59,5 & 1 & \\
\hline$>30$ anos & 155 & 36,9 & 33,3 & $1,06(0,94 ; 1,19)$ & 0,35 \\
\hline$<20$ anos & 30 & 7,1 & 7,2 & $1,02(0,81 ; 1,28)$ & 0,87 \\
\hline \multicolumn{6}{|l|}{ Estado marital } \\
\hline Com companheiro & 311 & 74,0 & 71,2 & 1 & \\
\hline Sem companheiro & 109 & 26,0 & 28,8 & $0,95(0,82 ; 1,07)$ & 0,44 \\
\hline \multicolumn{6}{|l|}{ Escolaridade } \\
\hline$\geq 9$ anos & 394 & 93,8 & 98,2 & 1 & \\
\hline$<9$ anos & 26 & 6,2 & 1,8 & $1,28(1,12 ; 1,45)$ & 0,01 \\
\hline \multicolumn{6}{|l|}{ Trabalho remunerado } \\
\hline Não & 276 & 65,7 & 66,7 & 1 & \\
\hline Sim & 144 & 34,3 & 33,3 & $1,01(0,90 ; 1,14)$ & 0,80 \\
\hline \multicolumn{6}{|l|}{ Renda familiar } \\
\hline$>2$ salários mínimos & 109 & 26,0 & 31,5 & 1 & \\
\hline$\leq 2$ salários mínimos & 311 & 74,0 & 68,5 & $1,11(0,86 ; 1,28)$ & 0,14 \\
\hline \multicolumn{6}{|l|}{ Realização do pré-natal } \\
\hline Sim & 413 & 98,3 & 100,0 & 1 & \\
\hline \multirow{2}{*}{\multicolumn{6}{|c|}{$\begin{array}{l}\text { Nao/Nao sabe } \\
\text { Local do pré-natal }\end{array}$}} \\
\hline & & & & & \\
\hline SUS & 358 & 86,7 & 93,7 & 1 & \\
\hline Plano de saúde particular & 55 & 13,3 & 6,3 & $1,23(1,09 ; 1,39)$ & 0,01 \\
\hline \multicolumn{6}{|c|}{ Características da criança } \\
\hline \multicolumn{6}{|l|}{ Idade da criança (anos) } \\
\hline$\geq 1$ ano & 302 & 71,7 & 75,7 & 1 & \\
\hline$\geq 6$ meses a 1 ano & 73 & 17,4 & 16,2 & $1,04(0,90 ; 1,21)$ & 0,57 \\
\hline$<6$ meses & 45 & 10,9 & 8,1 & $1,11(0,94 ; 1,30)$ & 0,21 \\
\hline \multicolumn{6}{|l|}{ Sexo da criança } \\
\hline Masculino & 220 & 52,4 & 54,1 & 1 & \\
\hline Feminino & 200 & 47,6 & 45,9 & $1,02(0,91 ; 1,15)$ & 0,68 \\
\hline \multicolumn{6}{|l|}{ Peso ao nascer } \\
\hline$\geq 2500$ gramas & 288 & 92,9 & 93,6 & 1 & \\
\hline$<2500$ gramas & 22 & 7,1 & 6,4 & $1,05(0,78 ; 1,42)$ & 0,72 \\
\hline Comprimento ao nascer & & & & & \\
\hline$\geq 47 \mathrm{~cm}$ & 274 & 93,5 & 95,5 & 1 & \\
\hline$<47 \mathrm{~cm}$ & 19 & 6,5 & 4,5 & $1,19(0,90 ; 1,59)$ & 0,22 \\
\hline Orientações & obre a & nportâr & cia da caderneta de saúde da criança & & \\
\hline Recebeu orientações sobre a CSC & & & & & \\
\hline $\operatorname{Sim}$ & 218 & 51,9 & 71,2 & 1 & \\
\hline Não & 202 & 48,1 & 28,8 & $1,32(1,17 ; 1,48)$ & 0,01 \\
\hline Profissionais que realizaram as orie & tações & & & & \\
\hline Enfermeiro & 151 & 69,3 & 75,9 & 1 & \\
\hline Outro profissional de nível superior & 53 & 24,3 & 19,0 & $1,19(0,96 ; 1,47)$ & 0,11 \\
\hline Profissional de nível médio & 14 & 6,4 & 5,1 & $1,18(0,83 ; 1,69)$ & 0,35 \\
\hline
\end{tabular}

Nota "Variável analisada apenas para os participantes que realizaram pré-natal $(n=413)$

"Variável analisada apenas para os participantes que receberam informações sobre a CSC ( $n=218)$

CSC = caderneta de saúde da criança; RP = Razão de prevalência; IC = Intervalo de confiança; SUS = Sistema único de saúde. 
Tabela II - Distribuição do percentual de preenchimento satisfatório da Caderneta de Saúde da Criança, conforme itens avaliados, Hospital Infantil Cosme e Damião. Porto Velho, Rondônia, Brasil, 2017 ( $n=420)$.

\begin{tabular}{|c|c|c|}
\hline Itens avaliados & $\mathbf{n}$ & $\%$ \\
\hline \multicolumn{3}{|l|}{ Dados sobre gravidez, parto e puerpério } \\
\hline Tipo do parto & 233 & 55,5 \\
\hline Dados do parto & 190 & 45,2 \\
\hline Dados da gravidez & 133 & 31,7 \\
\hline Dados do puerpério & 89 & 21,2 \\
\hline \multicolumn{3}{|l|}{ Dados do recém-nascido } \\
\hline Data do nascimento & 315 & 75,0 \\
\hline Peso ao nascer & 312 & 74,3 \\
\hline Local do nascimento & 306 & 72,9 \\
\hline Comprimento ao nascer & 293 & 69,8 \\
\hline Perímetro cefálico ao nascer & 273 & 65,0 \\
\hline APGAR & 272 & 64,8 \\
\hline Teste do pezinho & 82 & 19,5 \\
\hline \multicolumn{3}{|c|}{ Dados do monitoramento do crescimento e desenvolvimento da criança } \\
\hline Anotações de peso & 154 & 36,9 \\
\hline Anotações de comprimento por altura & 147 & 35,0 \\
\hline Curva de crescimento peso por idade & 120 & 28,6 \\
\hline Curva de crescimento comprimento por idade & 101 & 24,0 \\
\hline Curva de crescimento perímetro cefálico por idade & 92 & 21,9 \\
\hline Desenvolvimento neuropsicomotor & 202 & 18,1 \\
\hline \multicolumn{3}{|l|}{ Informações adicionais } \\
\hline Registro de vacinação & 417 & 99,3 \\
\hline Suplementação de vitamina A & 195 & 46,4 \\
\hline Registro de alimentação e problemas relacionados & 35 & 8,3 \\
\hline Registro de intercorrências & 14 & 3,3 \\
\hline
\end{tabular}

\section{DISCUSSÃO}

Os resultados do presente estudo permitiram identificar que a maioria das CSC tiveram preenchimento não satisfatório quando o cuidador principal não era os pais ou avós, possuíam escolaridade menor que nove anos, a mãe não havia realizado o pré-natal, ou não fez o acompanhamento pelo SUS, e entre os cuidadores que não receberam orientações sobre a importância da caderneta. O maior índice de preenchimento da caderneta foi referente às vacinas aplicadas, e um dos menores índices foi sobre o desenvolvimento neuropsicomotor.

A maioria dos cuidadores eram os próprios pais, destacando-se a presença materna. De forma geral e independente da classe social, a mãe é responsável pelo cuidado dos filhos, sobretudo nos primeiros anos de vidaa ${ }^{(14,18,24)}$. Portanto, esse é um papel social ainda "naturalizado" como tarefa unicamente feminina ${ }^{(24)}$, embora o lugar do pai como cuidador da criança esteja sofrendo mudanças substanciais e gradativas ${ }^{(25)}$.

No atual estudo, pouco mais da metade dos cuidadores receberam orientações sobre a CSC. Esse percentual foi um pouco acima dos $49,9 \%$ obtido em estudo no semi-árido brasileiro, no município de Feira de Santana $(\text { Bahia })^{(22)}$. Embora seja um direito universal das crianças nascidas em território brasileiro e sua distribuição seja gratuita, o desconhecimento da importância da CSC pelos pais e profissionais de saúde pode fragilizar o cuidado pela família à criança e inviabilizar a detecção precoce dos agravos prevalentes na infância, sobretudo pela falta de capacitação dos profissionais atuantes nos serviços da Atenção Primária em Saúde (APS) ${ }^{(9)}$.

A CSC é um prontuário infantil crucial no acompanhamento da saúde infantil, preconizado pela Política Nacional de Atenção Integral à Saúde da Criança (PNAISC) ${ }^{(8)}$. Nessa caderneta, os profissionais de saúde devem anotar os dados e eventos significativos referentes à criança, possibilitando um diálogo entre usuários/família e diversos profissionais $^{(12,17,18)}$ e estudantes da área de saúde ${ }^{(13)}$ que atendem a criança.

As orientações sobre a CSC aos cuidadores foram realizadas mais por enfermeiros $(69,3 \%)$ no presente estudo. O enfermeiro tem papel de destaque na organização cotidiana dos serviços e no contato com as crianças e seus cuidadores, favorecendo uma aliança entre profissional, usuário e família ${ }^{(11,15)}$. Uma investigação em João Pessoa, Paraíba, com 45 enfermeiros, sobre crescimento e desenvolvimento infantil, concluiu que os conhecimentos dos entrevistados sobre a temática se mostraram incipientes, um fato preocupante já que é um importante indicador 
para a vigilância da saúde infantil, teoricamente abordado na formação, e uma das atribuições cotidianas da prática de enfermeiros na APS ${ }^{(11)}$.

O percentual de preenchimento satisfatório das CSC avaliadas na presenta pesquisa foi muito baixo $(25,5 \%)$ e inferior aos $31,9 \%$ encontrado em cidades do estado do Piauí(22), indicando subutilização da CSC pelos profissionais de saúde. Por outro lado, neste estudo, o preenchimento insatisfatório da CSC esteve relacionado, entre outros aspectos, à baixa escolaridade do principal cuidador. O nível mais elevado de escolaridade dos cuidadores pode influenciar o padrão de crescimento e desenvolvimento nos primeiros anos de vida. Cuidadores mais escolarizados têm melhores condições de entender e assimilar as exigências de cuidados à saúde de seus filhos ${ }^{(3,21)}$.

No atual estudo, chama a atenção o baixo nível de preenchimento da CSC sobre a gravidez $(31,7 \%)$ e o puerpério $(21,2 \%)$. Um estudo em Cuiabá, Mato Grosso, que analisou os dados sobre gestação e parto/puerpério, também encontrou baixos índices de preenchimento. Esses registros são essenciais para avaliar, retrospectivamente, as condições de saúde da criança desde a gestação até o nascimento. Quando negligenciadas, podem influenciar de forma negativa a qualidade da assistência ao binômio mãe-filho ${ }^{(21,26)}$.

Outro aspecto preocupante na presente pesquisa é que apenas $65 \%$ das cadernetas possuíam registros sobre o perímetro cefálico ao nascimento. Os primeiros anos de vida são considerados críticos para o desenvolvimento das habilidades motoras, cognitivas e sensoriais. Doenças, como a microcefalia, podem afetar a maturação do sistema nervoso central e, quando não identificados/tratadas precocemente, podem comprometer em definitivo a criança ${ }^{(27)}$.

Em Rondônia, os registros referentes ao teste do pezinho (19,5\%) foram bem inferiores ao percentual de preenchimento encontrado nos demais itens avaliados na caderneta, o que permite uma reflexão sobre possíveis fragilidades no diagnóstico precoce de doenças graves, porém tratáveis. No município de Cuiabá, estado do Mato Grosso, em um estudo envolvendo 950 prontuários de crianças menores de um ano foi encontrado um percentual de preenchimento sobre o teste do pezinho de $14,1 \%{ }^{(21)}$, valor inferior ao encontrado na capital de Porto Velho, Rondônia. O teste do pezinho tem como finalidade rastrear precocemente doenças graves, como a fenilcetonúria, o hipotireoidismo congênito e a fibrose cística. Para essas doenças, o êxito do tratamento está na identificação nos primeiros meses de vida ${ }^{(28)}$.

O nível de preenchimento sobre o crescimento e desenvolvimento das crianças avaliadas também despertou preocupação no estudo em questão, posto que as anotações desses dois aspectos se resumiram à idade/peso e comprimento/altura, em detrimento aos escassos registros sobre curvas da idade/perímetro cefálico $(21,9 \%)$ e da avaliação dos marcos de desenvolvimento neuropsicomotor (18,1\%).

Em um dos estudos realizado no Nordeste do país ${ }^{(22)}$, encontrou-se uma frequência de $24,2 \%$ para o preenchimento do desenvolvimento neuropsicomotor, valor superior ao encontrado no estado de Rondônia. Os registros desses dados são cruciais para identificar/tratar precocemente graves problemas de saúde da criança. Os marcos do desenvolvimento infantil, registrados na CSC, são referências, por exemplo, para monitorar o desenvolvimento neuropsicomotor (DNPM) de crianças expostas a infecções, como zika, sífilis, toxoplasmose, citomegalovírus, herpes simplex e outras etiologias não infecciosas ${ }^{(27)}$.

Apesar de sua importância, o grau de preenchimento da CSC em Porto Velho, Rondônia, assim como em diferentes regiões do país, revela resultados desoladores. Além de ausências de registros, ocorrem falhas/ausências no preenchimento das curvas de crescimento e desenvolvimento na CSC ${ }^{(14,16,17)}$. Parece existir uma queda no nível de vigilância da saúde infantil à medida que a criança cresce. Em uma análise da CSC de 321 menores de cinco anos, de dois municípios da Paraíba, assistidos por equipes da Estratégia Saúde da Familia (ESF), todos os parâmetros tiveram falhas de registros, especialmente entre crianças com mais de 25 meses e entre aquelas que moravam em áreas com poucos profissionais nas equipes de saúde ${ }^{(21)}$.

Os registros sobre vacinação foram os mais completos (99,3\%), obtendo, mesmo assim, resultados inferiores aos $100 \%$ de preenchimento obtido em um estudo realizado em Pouso Alegre, Minas Gerais ${ }^{(16)}$. Os altos índices de registro de vacinação em relação aos demais pode ser atribuído à concepção da CSC como um "cartão de vacina", como parte das práticas cotidianas de saúde destinadas à mulher/criança na linha de cuidado ${ }^{(16)}$.

No atual estudo, a realização da quase totalidade dos registros de vacinação pode ser atribuída, em parte, aos investimentos de educação continuada dos profissionais da APS voltados, exclusivamente, ao manejo técnico da imunização, em detrimento a todos os demais itens da CSC. Até porque esses registros estão sob a responsabilidade da equipe de Enfermagem, que atua exclusivamente na sala de vacina, diferentemente dos outros itens, que são registrados por médicos, enfermeiros e outros profissionais em diferentes momentos e espaços assistenciais.

A pouca adesão dos profissionais a preencher os demais itens da CSC precisa ser revista urgentemente pela gestão da atenção à saúde da criança no município investigado, em razão deste exibir elevadas taxas de óbito infantil por causas evitáveis e redutíveis por adequada atenção à saúde da mulher na gestação e no parto, 
e ao recém-nascido ${ }^{(29)}$, o que mereceria, da gestão pública de saúde, o enfrentamento desse desafio por meio de ferramentas para o monitoramento do crescimento e desenvolvimento infantil, como a CSC.

Quando bem utilizada, a CSC torna-se um prontuário acessível, de fácil manuseio, que retém uma riqueza de informações sobre a criança e está sempre à mão dos cuidadores, o que permite registros de todos os profissionais que assistem a criança, além de favorecer o vínculo com família, comunidade e serviços, bem como o acompanhamento do crescimento e desenvolvimento integral, principalmente na primeira infância.

No Brasil, desde 2015, a orientação do preenchimento qualificado da CSC é um dos principais alvos da promoção à saúde da criança, instituída pela PNAISC no âmbito do Sistema Único de Saúde. Essa estratégia, aliada à promoção do aleitamento materno, sobretudo na primeira infância, visa reduzir a morbimortalidade e, consequentemente, promover o pleno desenvolvimento e crescimento da criança ${ }^{(8)}$. Em face desse desafio, é crucial que gestores da Atenção Básica invistam na qualificação e apoiem os serviços de atenção à saúde materno-infantil, com destaque à promoção do Desenvolvimento na Primeira Infância, com a utilização oportuna da CSC pelos profissionais, privilegiando seus conteúdos e orientações sobre a criança para sua família e a comunidade ${ }^{(12,17,22)}$.

Os resultados deste estudo devem ser interpretados no contexto da atenção secundária em saúde, pois os cuidadores foram interrogados em um serviço ambulatorial e hospitalar pediátrico. Nessas condições, a avaliação da CSC foi restrita aos responsáveis que a traziam consigo e às informações autodeclaradas dos cuidadores. Portanto, são passíveis do viés de memória no momento da entrevista. Em nível local, os resultados do estudo são inéditos e poderão auxiliar na compreensão dessa problemática na Atenção Básica. Novas pesquisas poderão explorar a percepção dos profissionais de saúde e cuidadores sobre a CSC e os motivos do seu baixo preenchimento em Porto Velho.

\section{CONCLUSÃO}

O preenchimento insatisfatório da Caderneta de Saúde da Criança mostra as fragilidades no acompanhamento do crescimento e desenvolvimento integral, principalmente na primeira infância, estando associado às crianças cujo cuidador principal possuía baixa escolaridade e não era os pais ou avós. Algumas condições, como a ausência de pré-natal ou a futura mãe não receber orientações sobre a CSC, dificultaram ainda mais a prática de registros pelos profissionais, especialmente sobre o desenvolvimento neuropsicomotor da criança, que foi quase inexistente neste estudo.

\section{CONTRIBUIÇÕES}

Jeanne Lúcia Gadelha Freitas, Priscilla Perez da Silva Pereira, Rayanne Cavalcante Nascimento, Tatiane Maciel Mendes e Águida Thomaz Santos contribuíram com a elaboração e delineamento do estudo, análise e interpretação de dados, e redação e/ou revisão do manuscrito; Kátia Fernanda Alves Moreira contribuiu com a elaboração e delineamento do estudo, análise e interpretação de dados, e revisão do manuscrito; Nathália Halax Orfão e Daniela Ferreira Borba Cavalcante contribuíram com a análise e interpretação de dados, e redação e/ou revisão do manuscrito.

\section{CONFLITOS DE INTERESSE}

Os autores declaram que o artigo não apresenta potenciais conflitos de interesse.

\section{REFERÊNCIAS}

1. Danzhen Y, Hug L, Ejdemyr S, Idele P, Hogan D, Mathers C, et al. Global, regional, and national levels and trends in under-5 mortality between 1990 and 2015, with scenario-based projections to 2030: a systematic analysis by the un Inter-Agency Group for Child Mortality Estimation. United Nations Inter-Agency Group for Child Mortality Estimation (UN IGME). Lancet [Internet]. 2015 [acesso em 2018 Mar 28];386:2275-86. Disponível em: http://www.thelancet.com/journals/lancet/article/PIIS0140-6736(15)00120-8/abstract.

2. Hidechika A, Miwa I, Akiko H, Rumiko A, Yasuyo O. Core factors promoting a continuum of care for maternal, newborn, and child health in Japan. Biosci Trends [Internet]. 2018 [acesso em 2018 Mar 30];12(1):1-6. Disponível em: https://www.jstage.jst.go.jp/article/bst/12/1/12_2017.01304/_pdf/-char/en

3. Caminha MFC, Silva SL, Lima MC, Azevedo PTACC, Figueira MCS, Batista M Filho. Vigilância do desenvolvimento infantil: análise da situação brasileira. Rev Paul Pediatr [Internet]. 2017 [acesso em 2018 Jan 13];35(1):102-9. Disponível em: http://www.scielo.br/pdf/rpp/v35n1/1984-0462-rpp-2017-35-1-00009.pdf 
4. United Nations Children's Fund. Report 2017: estimates developed by the UN Inter-agency Group for Child Mortality Estimation, 2017 [Internet]. 2017 [acesso em 2018 Mar 25]. Disponível em: https://www.unicef.org/ publications/index_101071.html

5. Ministério da Saúde (BR). DATASUS. Informações de Saúde - Estatísticas Vitais - Óbitos por causas evitáveis em menores de cinco anos [Internet]. 2016 [acesso em 2018 Fev 10]. Disponível em: http://tabnet. datasus.gov.br/cgi/deftohtm.exe?sim/cnv/evita10uf.def

6. The Lancet. Maternal and child nutrition: executive summary of The Lancet maternal and child nutrition series [Internet]. 2013 [acesso em 2018 Abr 6]. Disponível em: https://www.thelancet.com/series/maternal-and-childnutrition

7. World Health Organization. Essential nutrition actions: improving maternal, newborn, infant and young child health and nutrition [Internet]. 2013 [acesso em 2018 Abr 7]. Disponível em: http://www.who.int/nutrition/ publications/infantfeeding/essential_nutrition_actions/en/

8. Brasil. Ministério da Saúde. Portaria No 1.130, de 5 de agosto de 2015. Institui a Política Nacional de Atenção Integral à Saúde da Criança (PNAISC) no Âmbito do Sistema Único de Saúde (SUS) [Internet]. Diário Oficial da União; Brasília, 05 Ago. 2015 [acesso em 2018 Abr 10]. Disponível em: http://bvsms.saude.gov.br/bvs/ saudelegis/gm/2015/prt1130_05_08_2015.html

9. Lima LG, Nobre CS, Lopes ACMU, Rolim KMC, Albuquerque CM, Araújo MAL. A Utilização da caderneta de saúde da criança no acompanhamento infantil. Rev Bras Ciênc Saúde [Internet]. 2016 [acesso em 2018 Jan 12];20(2):167-74. Disponível em: http://www.periodicos.ufpb.br/ojs/index.php/rbcs/article/view/21266/15747

10. Osaki K, Hattori T, Toda A, Mulati E, Hermawan L, Pritasari K, et al. Maternal and Child Health Handbook use for maternal and child care: a cluster randomized controlled study in rural Java, Indonesia. J Public Health [Internet]. 2018 [acesso em 2018 Mar 26];40:1-13. Disponível em: https://academic.oup.com/jpubhealth/ advance-article/doi/10.1093/pubmed/fdx175/4793391 doi:10.1093/pubmed/fdx175

11. Reichert APS, Almeida AB, Souza LC, Silva MEA, Collet N. Vigilância do crescimento infantil: conhecimento e práticas de enfermeiros da atenção primária à saúde. Rev Rene [Internet]. 2012 [acesso em 2018 Abr 12];13(1)114-26. Disponível em: http://www.periodicos.ufc.br/rene/article/view/3780

12. Silva FB, Gaíva MAM. Preenchimento da caderneta de saúde da criança: percepção dos profissionais. Ciênc Cuid Saúde [Internet]. 2015 [acesso em 2018 Jan 3];14(2):1027-34. Disponível em: http://www.periodicos. uem.br/ojs/index.php/CiencCuidSaude/article/view/24268

13. Debs DHSL, Dias SR, Debs YD, Ferrão ACL, Carrijo JBC, Cualheta LP, et al. Avaliação do preenchimento da caderneta de saúde da criança após 4 anos da inserção do estudante de medicina no cenário prático. Rev Master [Internet]. 2016 [acesso em 2018 Abr 16];1(1):39-47. Disponível em: https://imepac.edu.br/ revistamaster/index.php/RM/issue/view/Revista\%20Master\%20v.1\%20n.1

14. Palombo CNT, Duarte LS, Fujimori E, Toriyama ATM. Use and records of child health handbook focused on growth and development. Rev Esc Enferm USP [Internet]. 2014 [acesso em 2018 Fev 16];48(Esp):59-66. Disponível em: http://www.revistas.usp.br/reeusp/article/view/103036/101315

15. Silva FB, Gaíva MAM, Mello DF. Utilização da caderneta de saúde da criança pela família: percepção dos profissionais. Texto \& Contexto Enferm [Internet]. 2015 [acesso em 2018 Abr 16];24(2):407-14. Disponível em: http://www.scielo.br/scielo.php?pid=S0104-07072015000200407\&script=sci_arttext\&tlng=pt

16. Faria M, Nogueira TA. Avaliação do uso da Caderneta de Saúde da Criança nas Unidades Básicas de Saúde em um município de Minas Gerais. Rev Bras Ciênc Saúde [Internet]. 2013 [acesso em 2017 Dez 16];11(38):8-15. Disponível em: http://seer.uscs.edu.br/index.php/revista_ciencias_saude/article/ view/1944/1469

17. Almeida AC, Mendes LC, Sad IR, Ramos EGA, Fonseca VM, Peixoto MVM. Uso de instrumento de acompanhamento do crescimento e desenvolvimento da criança no Brasil-Revisão sistemática de literatura. Rev Paul Pediatr [Internet]. 2016 [acesso em 2018 Out 09];34(1):122-31. Disponível em: http://www.scielo.br/ $\mathrm{pdf} / \mathrm{rpp} / \mathrm{v34n1/pt \_ 0103-0582-rpp-34-01-0122.pdf}$

18. Amorim LP, Senna MIB, Gomes VE, Amaral JHL, Vasconcelos M, Silva AG, et al. Preenchimento da Caderneta de Saúde da Criança nos serviços de saúde em Belo Horizonte, Minas Gerais, Brasil. Epidemiol 
Serv Saude Brasília [Internet]. 2018 [acesso em 2018 Fev 15];27(1):e201701116. Disponível em: http://www. scielo.br/pdf/ress/v27n1/2237-9622-ress-27-01-e201701116.pdf

19. Ministério da Saúde (BR). DATASUS. População residente por Faixa Etária 1 segundo Região de Saúde (CIR) Período: 2015 [Internet]. 2015 [acesso em 2017 Nov 23]. Disponível em: http://tabnet.datasus.gov.br/ cgi/tabcgi.exe?novapop/cnv/popbr.def

20. Secretaria de Estado da Saúde (RO). SESAU: relatório de gestão 2015: resultados e perspectivas. Porto Velho: Governo do Estado de Rondônia; 2016.

21. Abud SM, Gaíva MAM. Registro dos dados de crescimento e desenvolvimento na caderneta de saúde da criança. Rev Gaúcha Enferm [Internet]. 2015 [acesso em 2017 Out 27];36(2):97-105. Disponível em: http:// www.scielo.br/scielo.php?pid=S1983-14472015000200097\&script=sci_arttext\&tlng=pt

22. Costa JSD, Cesar JA, Pattussi MP, Fontoura LP, Barazzetti L, Nunes MF, et al. Assistência à criança: preenchimento da caderneta de saúde em municípios do semi-árido brasileiro. Rev Bras Saúde Matern Infant [Internet]. 2014 [acesso em 2017 Nov 23];14(3):219-27. Disponível em: http://www.scielo.br/pdf/rbsmi/ v14n3/1519-3829-rbsmi-14-03-0219.pdf

23. Brasil. Ministério da Saúde, Conselho Nacional de Saúde. Resolução nº. 466, de 12 de dezembro de 2012 [Internet]. 2002 [acesso em 2018 Abr 10]. Disponível em: http://bvsms.saude.gov.br/bvs/saudelegis/cns/2013/ res0466_12_12_2012.html

24. Barreta C, Oliveira MAM, Dias AM, Chesant FH. Caracterização dos cuidadores das crianças e dos adolescentes atendidos pelo projeto de extensão de um hospital universitário infantil. Rev Bras Tec Sociais [Internet]. 2016 [acesso em 2017 Nov 21];14(3):219-27. Disponível em: https://siaiap32.univali.br/seer/index. php/rbts/article/view/9746/5478

25. Arruda SLS, Lima MCF. O novo lugar do pai como cuidador da criança. Estud Interdiscip Psicol [Internet]. 2013 [acesso em 2017 Oct 13];4(2):201-16. Disponível em: http://pepsic.bvsalud.org/pdf/eip/v4n2/a06.pdf

26. Gaíva MAM, Silva FB. Caderneta de saúde da criança: revisão integrativa. Rev Enferm UFPE on line [Internet]. 2014 [acesso em 2017 Set 24];742-9. Disponível em: https://periodicos.ufpe.br/revistas/ revistaenfermagem/article/viewFile/9733/9830

27. Ministério da Saúde (BR), Secretaria de Vigilância em Saúde. Secretaria de Atenção à Saúde. Orientações integradas de vigilância e atenção à saúde no âmbito da Emergência de Saúde Pública de Importância Nacional: procedimentos para o monitoramento das alterações no crescimento e desenvolvimento a partir da gestação até a primeira infância, relacionadas à infecção pelo vírus Zika e outras etiologias infecciosas dentro da capacidade operacional do SUS [Internet]. 2017 [acesso em 2018 Set 10]. Disponível em: http:// portalarquivos.saude.gov.br/images/pdf/2016/dezembro/12/orientacoes-integradas-vigilancia-atencao.pdf

28. Oliveira EF, Souza AP. Importância da realização precoce do teste do pezinho: o papel do enfermeiro na orientação da triagem neonatal. Id on Line Rev Psic [Internet]. 2017 [acesso em 2017 Set 28];11(35):361-78. Disponível em: https://idonline.emnuvens.com.br/id/article/view/742/1037

29. Moreira KFA, Oliveira TS, Gonçalves TA, Moura CO, Maluf SN, Tavares RSA, et al. Mortalidade infantil nos últimos quinquênios em Porto Velho, Rondônia - Brasil. Rev Bras Crescimento Desenvolv Hum [Internet] 2014 [acesso em 2017 Ago 30];24(1):86-92. Disponível em: http://www.revistas.usp.br/jhgd/article/ view/76123/79880

\author{
Endereço para correspondência: \\ Jeanne Lúcia Gadelha Freitas \\ Fundação Universidade Federal de Rondônia - UNIR \\ BR 364, Km 9,5 \\ CEP: 76801-059 - Porto Velho - RO - Brasil \\ E-mail: jeannegadelha@unir.br
}

Como citar: Freitas JLG, Pereira PPS, Moreira KFA, Orfão NH, Cavalcante DFB, Nascimento RC, et al. Preenchimento da caderneta de saúde da criança na primeira infância. Rev Bras Promoç Saúde. 2019;32:8407. 